\title{
DIE SCHWEIZERISCHE LICHTBILDANSTALT UND DIE GEOGRAPHIE
}

\author{
Von Max Gschwend
}

Seit Jahrzehnten hat das Lichtbild im geographischen Unterricht und in der geographischen Forschung in stets zunehmendem Maße an Bedeutung gewonnen. Jeder, der im Unterricht steht, sei es an der Mittelschule oder an der Universität, kennt dieses Hilfsmittel und benützt es reichlich. Aus dem wissenschaftlichen Leben ist das Lichtbild nicht mehr wegzudenken. Zu groß sind die Vorteile, die ein gutes, klares und technisch einwandfreies Lichtbild dem Lehrer bietet: beim Darstellen eines Begriffes, einer unbekannten Region, oder um die Schüler zum selbständigen Beobachten und Erarbeiten zu erziehen. Besonders für die Geographie ist es wichtig - ganz speziell dann, wenn für die Schüler die eigene persönliche Anschauung in der Natur fehlt daß durch das Bild ein möglichst guter Ersatz geboten wird. Hier steht im Zentrum des Unterrichts wie nirgends sonst: "Die Grundlage aller Erkenntnis ist die Anschauung. » Erzählungen, Beschreibungen, auch in dichterischer Form, vermögen nicht mit derselben Eindrücklichkeit einen Menschen, sei er nun Schüler oder Erwachsener, zu packen wie das Bild. Unterricht am Lichtbild jedoch bietet noch mehr. Der Lichtkegel, der das Dunkel durchbricht, zwingt die Aufmerksamkeit eines jeden auf das Bild an der Leinwand. Störende Begleittexte fehlen. Nur das Wort des Lehrers gibt den Schülern einen Hinweis, und bereits setzt spontan, gefördert durch reges Interesse, ein lebhaftes Besprechen der gebotenen Lichtbilder ein, was dem Lehrer erlaubt, durch einfache Hinweise die Richtung und die Tiefe der Verarbeitung zu dirigieren.

Es ist selbstverständlich, daß die erfolgreiche Verwendung des Lichtbildes einwandfreies Bildmaterial voraussetzt. Einwandfrei ist das Bildgut aber nur dann, wenn es nicht nur sachlich, inhaltlich das bietet, was man im gegebenen Fall darstellen oder zeigen will, sondern wenn es auch technisch hervorragend ist, d. h. sauber, klar und scharf. Diese Anforderungen, die an das Lichtbild gestellt werden müssen, soll es seinem Zweck genügen, sind jedoch nicht leicht und ohne weiteres zu erfüllen. Es braucht hiezu eine Zentrale, welche Bildmaterial aus allen Kulturgebieten sammelt, sichtet und ausscheidet, veraltetes Material durch neues ersetzt und es in übersichtlicher Form (Bildkartothek) den Interessenten darbietet und die technisch einwandfreie Herstellung der Lichtbilder gewährleistet.

Diesen Erkenntnissen, die zum Teil in anderen Staaten schon seit Jahren verwirklicht wurden, auch in der Schweiz zum Durchbruch verholfen zu haben, ist das Verdienst von Ingenieur LEO WYRSCH (Zürich), dessen Initiative es zu verdanken ist, $\mathrm{da}$ sich die interessierten Kreise und Verbände in der "Schweizerischen Arbeitsgemeinschaft für das Lichtbild» zusammenschlossen.

Als Zentralstelle wurde 1941 die Schweizerische Lichtbildanstalt in Zürich gegründet. $\mathrm{Daß}$ diese Gründung einem großen Bedürfnis weiter Kreise aus Industrie, Technik, Wirtschaft, Wissenschaft und Unterricht entsprach, zeigte die Mitarbeit, das Entgegenkommen und die Hilfe, die besonders in den ersten Jahren die finanzielle Grundlage der neuen Anstalt sicherten. Die Leitung lag in den Händen des Geschäftsführers L. WYRSCH, dem Prof. Dr. E. RÜsT vom Photographischen Institut der ETH. zur Seite stand.

Die wichtigste Aufgabe der Schweizerischen Lichtbildanstalt besteht im Sammeln und gebrauchsmäßigen Ordnen von Bildgut aus Natur, Technik, Kunst und Kultur für Wissenschaft, Unterricht und Vortrag. Dabei ist unter Lichtbild jedes durch photographisches Verfahren erzeugte Bild zu verstehen (Negative, Papierbilder, Diapositive, Filme usw.). 
Des weiteren sollen durch das neugeschaffene Dokumentationsmittel der Sch weizer Bildkarte im Laufe der Zeit an verschiedenen Stellen des Landes Kartotheken entstehen, die über das von der Lichtbildanstalt gesammelte Bildmaterial Auskunft geben. Die Bildkarte bietet das mit kurzem Text und Kennummer versehene photographische Bild und ermöglicht eine allmähliche Erfassung des Bildbestandes. Die Aufstellung an verschiedenen Orten und die Ordnung dieser Bildkarten nach verschiedensten Gesichtspunkten ermöglichen eine intensive Auswertung. Die Lichtbildanstalt ist durch ihre technischen Einrichtungen befähigt, die gewünschten Bilder in jeder verlangten Form herzustellen, da sie stets die erforderlichen Bildträger für weitere Vervielfältigungen zur Verfügung hat oder erhalten kann. Bedarfspläne für die Verwendung des Lichtbildes in den verschiedensten Sektoren sollen das notwendige Bildgut erfassen und gliedern. Richtlinien beleuchten die zweckmäßige Gestaltung und Verwendung des Lichtbildes und des Unterrichtsfilmes.

Die Zentralstelle fördert die Zusammenarbeit aller am Lichtbild interessierten Kreise, u. a. durch Gründung von Arbeitsgemeinschaften (z. B. Schweizerische Arbeitsgemeinschaft für das Lichtbild, Arbeitsgemeinschaft Schweizer Filmhaus, Arbeitsgemeinschaft für photomechanische Industrie).

Eine wichtige Aufgabe der Schweizerischen Lichtbildanstalt ist die gewissenhafte Beratung in allen Fragen, die das Lichtbild und seine Anwendung betreffen. Sie gibt Auskunft über vorhandene Bildbestände, die zweckmäßigen Formen der Bilddarbietung, die inhaltliche, technische und zeichnerische Bildgestaltung und die Neuschaffung von Bildgut, ferner über die geeigneten Geräte, die Einrichtung des Bildwurfraumes und alle photographischen Dokumentierungsarten. Durch geeignete Vorführungen fördert sie die Verwendung des Lichtbildes und hilft durch Aufklärung in Presse und Publikationen mit, daß das gute und wahre Bild in vermehrtem Maß zur Verständigung herangezogen wird. Insbesondere setzt sich die Lichtbildanstalt für die zweckmäßige Aufbewahrung und Ordnung der photographischen Negative ein, gleichgültig, ob sie sich in ihrem Besitz oder in Archiven, Museen, Bibliotheken, Werken und bei Photographen befinden. Eine planmäßige Lenkung des archivmäßigen Sammelns photographischer Urkunden gestattet, wertvolle Aufnahmen zu erhalten, bekanntzumachen und praktisch zu verwerten. Die Aufstellung einer Leihsammlung von Lichtbildern und Filmen wird nach Bedarf und entsprechend den vorhandenen Mitteln ausgebaut.

Die Wichtigkeit und die große kulturelle Aufgabe des Institutes und die Notwendigkeit, den Sinn für lebensechte und in allen Beziehungen gute Bilder zu wecken und zu fördern, übersteigen weit die Kräfte eines gewöhnlichen kaufmännischen Reproduktionsunternehmens. Es ist daher notwendig, daß Eidgenossenschaft und Kantone durch vermehrte Zuwendungen der Lichtbildanstalt ermöglichen, ihre im gesamtschweizerischen Interesse liegenden kulturellen Aufgaben zu lösen. Kulturelle Arbeiten können sich nie selber finanzieren und sind überall auf staatliche Unterstützungen angewiesen. So ist auch die Lichtbildanstalt kaum in der Lage, ihre eigentlichen Aufgaben zu lösen, obwohl der Betrieb so wirtschaftlich wie möglich gestaltet wurde. Insbesondere die verantwortungsvollen Probleme, die für Schule, Unterricht und das kulturelle Leben der Schweiz von Bedeutung sind, können nicht mit ungenügenden Kräften in Angriff genommen werden. Das bisherige schweizerische Kulturleben darf auch hier ausgezeichnete Qualitätsarbeit verlangen. Nur die Schweizerische Lichtbildanstalt wird dank ihren vorzüglichen technischen Einrichtungen in der Lage sein, diese Arbeiten auszuführen, Lichtbilder und Lichtbildreihen zu Preisen herzustellen, die auch für die Unterrichtsanstalten tragbar sind.

Nach Jahren intensiver Arbeit und begünstigt durch eine mustergültige Einrichtung hat die Schweizerische Lichtbildanstalt ihre Daseinsberechtigung bewiesen; die vielseitige Inanspruchnahme, die zahlreichen Besuche und Anfragen zeigen, daß sie einem Bedürfnis entspricht, wie denn auch ihr Aufgabenkreis etwas Einzigartiges in der 
Schweiz darstellt. Bei der in unserem Staate leider sprichwörtlichen sparsamen Einstellung gegenüber rein wissenschaftlichen und kulturellen Belangen, war es jedoch nicht möglich, das reiche Bildmaterial, das sich im Laufe der Jahre ansammelte (zu erwähnen sind vor allem die großen Negativsammlungen von Prof. Dr. A. Herm, von Prof. Dr. Wehrli, von Dr. Hirschi, von Christian und Hans Meisser sowie Sammlungen über schweizerische Baudenkmäler, Bürgerhäuser, Kunst usw.), in wünschenswerter Form auszuwerten und zugänglich zu machen. Immerhin konnte aus dem vorhandenen Material eine größere Anzahl von Lichtbildreihen, die eine thematisch geschlossene Einheit bilden, aus verschiedenen Gebieten, insbesondere der Technik, Industrie, Kunst und Geschichte, zusammengestellt und ausgearbeitet werden. Es wird eine der großen Aufgaben der Lichtbildanstalt sein, den Ausbau dieser Lichtbildserien weiter zu fördern und auf alle Wissensgebiete auszudehnen.

Die stets wachsende Bedeutung, die der Film für Unterricht und Wissenschaft gewinnt, führte im Herbst 1945 zur Gründung einer Arbeitsgemeinschaft Schweizer Filmhaus, die die Sammlung und den Verleih von Industrie- und Dokumentarfilmen bezweckt. Gemeinsam mit der Schweizerischen Arbeitsgemeinschaft für Unterrichtskinematographie (SAFU), deren Filmarchiv durch die Lichtbildanstalt verwaltet werden soll, werden Berufsfilme für Schulungszwecke herausgegeben. Besondere Aufmerksamkeit wird dem gezeichneten Lichtbild gewidmet. Es bietet in seiner abstrakten Form die Möglichkeit, wesentliche Teile hervorzuheben, durch Schematisierung zu vereinfachen unḍ damit zu klären. Insbesondere technische Lichtbildreihen eignen sich für diesen Zweck ganz gut. Selbstverständlich werden sie stets durch naturgetreue Lichtbilder ergänzt. Auch die Illustration von zahlreichen Lehrmitteln behilft sich gerne mit dem gezeichneten Bild ("Geographiebuch für Sekundarschulen» des Kantonalen Lehrmittelverlages, Zürich; "Fundamentation und Konsolidation» von Ing. Dr. KollbrunNer).

Wie ersichtlich, hat die Schweizerische Lichtbildanstalt eine weit über ihren engeren Rahmen hinausgehende kulturelle Aufgabe. Es soll ja hier eine zentrale Sammel- und Verwertungsstelle entstehen, die die vielseitigsten Wünsche, Bilder betreffend, befriedigen kann. Insbesondere hat sie für die Geographie und den geographischen Unterricht an sämtlichen Schulstufen der Schweiz eine ganz spezielle Mission. Das von allen Seiten zuströmende, ständig sich erneuernde Bildmaterial erlaubt einen hervorragenden Grundstock von einwandfreien, sachlich und ästhetisch befriedigenden Bildern zur allseitigen Verwendung bereitzustellen und Lichtbildsammlungen aufzubauen, die sachlich am besten. den Anforderungen verschiedener Bedürfnisse entsprechen ( $z$. B. Sammlungen für Volksschulen, für Mittel- und Hochschulen, für Vorträge usw.). Insbesondere wird es auch möglich sein, durch die wissenschaftliche Verarbeitung thematisch geschlossene geographische Lichtbildreihen, bestehend aus 20 bis 30 Stück, herauszugeben (wie das in anderen Wissensgebieten bereits geschah), begleitet von einem kurzen sachlichen Erläuterungstext, der auch dem Nichtfachmann die Verwendung dieser Reihen im Unterricht ermöglicht. Selbstverständlich können im Einzelfall solche Reihen durch Weglassen oder Umstellung von einzelnen Bildern auch den besonderen Bedürfnissen angepaßt werden. Diese Reihen enthalten "Kernbilder», das sind die wichtigsten, unbedingt erforderlichen zur Behandlung des Themas; Ergänzungsbilder zeigen weitere instruktive Besonderheiten, während Erweiterungsbilder auch weitergehenden Anforderungen genügen wollen und auf Spezialfälle hinweisen. Jedes Bild erhält eine klare Anschrift und einen kurzen Erläuterungstext. Der Kommentar gibt in einer knappen Behandlung die wichtigsten Angaben zum Thema und in einem Ergänzungstext weitere Notizen zu den einzelnen Bildern.

Neben diesen, nach sachlichen Gesichtspunkten abgerundeten Serien ist die Aufstellung von Typensammlungen für jedes einzelne Land und entsprechend auch für Kontinente für die Geographie wesentlich. Bereits ausgearbeitete Bedarfspläne zeigen in großen Zügen, was gesammelt werden soll und wie es zu ordnen ist. Diese Typen- 
sammlung, die die natürlichen und kulturellen Faktoren der Landschaftsgestaltung umfaßt, erlaubt ohne weiteres eine parallele Sammlung nach regionalen Gesichtspunkten, wobei graphische und kartographische Darstellungen mitberücksichtigt werden.

Auf die weittragende Bedeutung dieser Sammlungen, vor allem in geographischen Belangen, soll nur noch skizzenhaft hingewiesen werden. Welch reiche Möglichkeiten liegen darin für Wissenschaft, Schulung und Vorträge! Das Bildmaterial bietet die wertvollsten Unterlagen für Regional- und Landesplanung, die damit weitgehend gefördert werden. Auch die Werbung für den Fremdenverkehr und Tourismus findet hier ausgezeichnetes Material. Die nationale Kultur wird vertieft und erweitert und die allgemeine Bildung gefördert durch richtige Erziehung des Bildgeschmackes am guten Bild.

Die durchorganisierte Verbindungsarbeit mit allen bedeutenden Institutionen unseres Landes (z. B. Schweizerischer Geographielehrerverein, Schweizer Schullichtbild usw.) und die Mitarbeit der verschiedensten Kreise und zahlreicher Wissenschafter, Dozenten und Lehrer sowie von Fachleuten aus Industrie, Technik und Wirtschaft ermöglichen einen weiten Einblick und Überblick im vielseitigen Aufgabenkreis. Die allseitige Fühlungnahme gestattet der Schweizerischen Lichtbildanstalt, auf dem gesamten Gebiet des Lichtbildes anregend und fördernd zu wirken. Damit sucht sie die Forderung unserer kulturellen und nationalen Selbstbehauptung zu erfüllen, stets und überall nur beste Bilder schweizerischen Schaffens und schweizerischen Wesens zu zeigen.

\section{DIE SAMMLUNG FƯR VÖLKERKUNDE DER UNIVERSITATT ZURICH IM JAHRE 1946/47}

Im Zusammenhang mit Schenkungen und Leihgaben wurden in den Sammlungsräumen verschiedene Umgruppierungen der Schaukästen und Fenstervitrinen vorgenommen. Für die Aufnahme eines wertvollen, vom Museum Schaffhausen als Leihgabe überwiesenen Indianerkostüms mußten der Nordamerika-Schrank umgestellt und für die von Dr. H. SснӓRER (Borneo) zur Verfügung gestellten Objekte die Dayak-Vitrine neu geordnet werden. Neu eingerichtet wurden ferner eine kleine Fenstervitrine mit Luristanbronzen und prähistorischen Töpfereien aus dem vorderen Orient und eine Pultvitrine mit Silber und Jadeschmuck aus China. Neun Schränke der Südsee-Abteilung erfuhren eine Lockerung und Umgruppierung; überdies wurden die vom Unterzeichneten in Holland angekauften hindujavanischen Bronzen in einem Schaukasten übersichtlich ausgestellt.

Im Berichtsjahre wurde die Sichtung und Katalogisierung der als Dauerdepot überwiesenen Sammlungen und der Bibliothek von Prof. C. Täuber beendigt; ebenso wurde der Stichwortkatalog der wissenschaftlichen Handbibliothek fertiggestellt. Die systematische Katalogisierung derselben, wofür vom Kantonalen Arbeitsamt Max Huber (vom 10. X. 1945 bis 18. IV. 1946), JAKob RitTER (vom 10. IX. 1945 bis 10. III. 1946) und PAUL MORY (vom 28. III. bis 28. IX. 1946) vorübergehend zur Verfügung standen, die zugleich als Arbeitskräfte in der Sammlung für Völkerkunde tätig waren, konnte im Berichtsjahre noch nicht völlig zu Ende geführt werden. Von der Konservatorin, Frl. E. Leuzinger, wurde eine chronoptische Zeittafel, die als Úbersicht über Beginn und Dauer der einzelnen Hochkulturen der Erde und als Vergleich mit der europäischen Prähistorie und Geschichte dienen soll, zusammengestellt. Außerdem hat Frl. Leuzingrer eine für Schulen und Museumsbesucher bestimmte, in gedrängter Form zusammengefaßte allgemeine Wegleitung durch die Völkerkundesammlung, nach welcher schon lange ein Bedürfnis bestand, ausgearbeitet, die voraussichtlich im nächsten

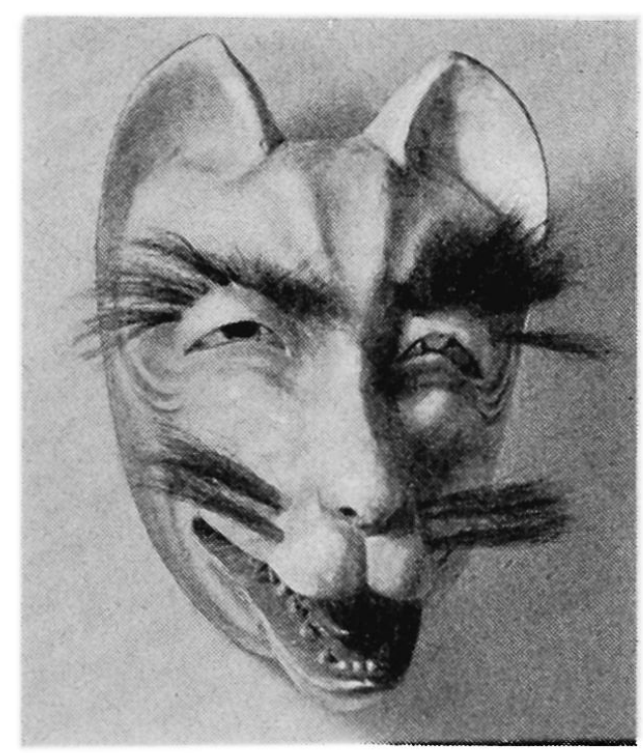

Abb. 1. Japanische Fuchsmaskc; Holz, lackicrt, mit cingesctztem Haar und beweglichem Unterkiefer. Der Fuchs spielt im Volksglauben und in der Mythologic der Japancr cinc bedcutende Rollc. Eine besondere Art dämonischer Besessenheit, dic besonders Fraucn befallen soll, wird mit ihm in Zusammenhang gebracht. Vor allem abcr ist der Fuchs mit dem Rciskult verknüpft, indem die Reisgöttin Inari in der Gestalt eines wcißen Fuchses dargestellt wird. Photo E. Leczivger 